Original article

\title{
Synthesis and antimicrobial studies on novel sulfonamides containing 4-azidomethyl coumarin
}

\author{
Mahantesha Basanagouda ${ }^{\mathrm{a}}$, K. Shivashankar ${ }^{\mathrm{b}}$, Manohar V. Kulkarni ${ }^{\mathrm{a}, *}$, Vijaykumar P. Rasal ${ }^{\mathrm{c}}$, \\ Harishchandra Patel ${ }^{c}$, Sumit S. Mutha ${ }^{c}$, Ashwini A. Mohite ${ }^{\mathrm{c}}$ \\ a P.G. Department of Chemistry, Karnatak University, Dharwad 580 003, Karnataka, India \\ ${ }^{\mathrm{b}}$ P.G. Department of Chemistry, Central College, Bangalore University, Bangalore 560 001, Karnataka, India \\ ${ }^{\mathrm{c}}$ Department of Pharmacology, KLES's College of Pharmacy, Belgaum-590 010, Karnataka, India
}

\section{A R T I C L E I N F O}

\section{Article history:}

Received 5 January 2009

Received in revised form

9 October 2009

Accepted 10 December 2009

Available online 21 December 2009

\section{Keywords:}

Coumarin

Sulfonamide

Azide

Antimicrobial activity

\begin{abstract}
A B S T R A C T
A series of new and novel coumarin-6-sulfonamides with a free C4-azidomethyl group have been synthesized as antimicrobials in three steps starting from 7-methyl-4-bromomethylcoumarin $\mathbf{1}$. The reaction of $\mathbf{1}$ with chlorosulfonic acid was found to yield the corresponding 6-sulfonylchloride $\mathbf{2}$, which when treated with sodium azide led to intermediate $\mathbf{3}$. The title sulfonamides $\mathbf{5 a}-\mathbf{y}$ were obtained from the reaction of $\mathbf{3}$ with various aromatic amines $\mathbf{4}$ in refluxing benzene. The chemical structures of the compounds were elucidated by IR, NMR and LC-MS spectral data. All the synthesized compounds have been screened for their in vitro anti-bacterial and anti-fungal activities. Some of the compounds have been found to be active against both bacterial species at a concentration of $1 \mu \mathrm{g} / \mathrm{mL}$.
\end{abstract}

(c) 2009 Elsevier Masson SAS. All rights reserved.

\section{Introduction}

Coumarins are a class of lactones which are found widely in nature and possess antiinflammatory [1,2], antiviral [3], antiHIV [4], antifungal [5] and cytotoxic activities [6,7], which have been reviewed [8]. In the development of newer antimicrobials, coumarins have been identified as target specific plant anti-bacterial agents with growth inhibitory potential particularly against Gram-positive species [9]. This is based on the related antibiotic novobiocin which targets the DNA gyrase [10]. Synthetic coumarins with chloramphenicol [11] side chain and cephalosporin amides [12] at C-3 position, have been screened against a number of bacterial species. One of the earliest attempts to enhance their antimicrobial potency led to 6sulphonamido coumarins [13-16], as anti-tubercular agents. Introduction of a basic side chain at the allylic position with respect to the biogenetically important C3-C4 double bond [17] was attempted to reduce the acidic character of coumarin antibiotics. Amongst the various alkyl amines and sulfides synthesized, the 4-piperazinomethyl and imidazomethyl sulfides [18] were found to be the most active compounds. We have been studying structurally analogous, 4-arylaminomethyl [19] 4-sulphonamidomethyl [20] and 4-

\footnotetext{
* Corresponding author. Tel.: +91 836 2215286; fax: + 918362747884

E-mail address: manohar274@gmail.com (M.V. Kulkarni).
}

dichloroacetamidomethyl coumarins [21] for their anti-bacterial activity. Recent reports on the cytotoxic effects of coumarin sulfonamides [22,23], on leukemia cell lines have generated a renewed interest in such class of compounds. Azido group has been recognized as a bioorthogonal chemical reporter which is stable in physiological conditions [24] and has been employed in the study of biosynthetic pathways of biomolecules $[25,26]$. It is also expected that the polar nature of the azide would play an important role in penetrating the cell wall thus enhancing the bacterial potency of the compounds. In continuation of our interest in designing oxygen heterocycle based anti-bacterials [27], we have synthesized new sulfonamides with an azido group at the allylic position in the coumarin ring and the results of anti-bacterial and anti-fungal activity have been discussed in this paper.

\section{Chemistry}

4-Bromoethylacetoacetate obtained from the bromination of ethylacetoacetate, was treated with $m$-cresol under Pechmann cyclization conditions using neat sulphuric acid as the condensing agent. The reaction resulted in the formation of 4-bromomethyl7-methylcoumarin $\mathbf{1}$. The reactivity of this compound has been explored in a cascaded manner via a regioselective electrophilic substitution, allylic $S_{\mathrm{N}}$ reaction and a nucleophilic displacement at the oxidized sulfur. Compound 1 was refluxed with excess of 
chlorosulfonic acid to give 4-bromomethyl-7-methyl-coumarin-6sulfonylchloride 2 . The regioselective electrophilic substitution at $\mathrm{C}-6$ is probably because of the electron donating $\mathrm{C} 7-\mathrm{CH}_{3}$ group and the spatial proximity of $\mathrm{C}-6$ with the ring oxygen, which resembles the para-position of phenols.

Treatment $\mathbf{2}$ with sodium azide in aqueous acetone at room temperature resulted in the formation of 4-azidomethyl-7-methylcoumarin-6-sulfonyl chloride $\mathbf{3}$, the structure of which was supported by spectral data. Preferential attack of the linear azide nucleophile at the allylic carbon is favorably due to the better leaving group ability of bromine and slight steric hindrance caused by the ortho-methyl group at C-7. However use of two equivalents of sodium azide at room temperature leads to the formation of bisazide expected because of the higher reactivity of sulfonyl chloride which has been recently reported in the reactions of 4-bromomethyl-benzenesulfonyl chloride [28]. Sulfonamides 5a-y were obtained by refluxing aromatic amines and sulfonyl chloride derivative $\mathbf{3}$ in dry benzene. Removal of solvent under reduced pressure afforded the title compounds as solids which were purified by routine methods (Scheme 1 ).

\section{Results and discussion}

Structure of C-6 sulfonyl chloride 2 was supported by the appearance of two singlets in the ${ }^{1} \mathrm{H}$ NMR at 7.40 and $8.45 \mathrm{ppm}$ due to $\mathrm{C} 5-\mathrm{H}$ and $\mathrm{C} 8-\mathrm{H}$ respectively. The $\mathrm{C}_{4}-\mathrm{CH}_{2}$ protons appeared as singlet at $4.52 \mathrm{ppm}$ where as $\mathrm{C}_{3}-\mathrm{H}$ observed as singlet at $6.63 \mathrm{ppm}$. Formation of azide 3 was indicated by a strong band at $2135 \mathrm{~cm}^{-1}$. It was the change in the chemical shift of methylene protons to $5.00 \mathrm{ppm}$ which supported the observed regiochemistry of the nucleophilic displacement at the allylic carbon under the present experimental conditions. Expectedly there was no significant change in the aromatic region with singlets appearing at 7.69 and $8.24 \mathrm{ppm}$. Generation of sulfonamides $\mathbf{5 a - y}$ was indicated by a sharp band around $3360 \mathrm{~cm}^{-1}$ and the azide group remained in-tact during the nucleophilic displacement at the sulfonyl chloride 3 . In the PMR spectrum of 4-azidomethyl-7-methyl-coumarin-6sulfonic acid $p$-tolylamide (5c) $\left(\mathrm{R}=-\mathrm{C}_{6} \mathrm{H}_{4}-\mathrm{CH}_{3}-p\right)$ showed two singlets at 2.29 and $2.66 \delta$ ppm due to $\mathrm{C} 7-\mathrm{CH}_{3}$ and $\mathrm{CH}_{3}$ of phenyl ring, respectively. The $\mathrm{NH}$ proton was observed as singlet at $4.49 \mathrm{ppm}$. The methylene protons linked to azido group were observed as<smiles>Cc1cccc(O)c1</smiles><smiles>CCOC(=O)CC(=O)CBr</smiles><smiles>Cc1cc2oc(=O)cc(CN)c2cc1S(=O)(=O)Cl</smiles><smiles>CC(C)(O)[Mg][Mg]</smiles>

3

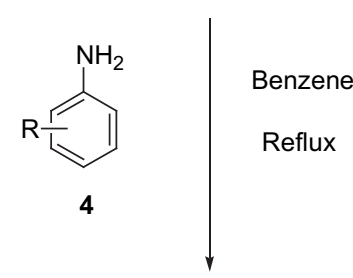<smiles>[R][X]c1ccc(NS(=O)(=O)c2cc3c(CN)cc(=O)oc3cc2C)cc1</smiles>

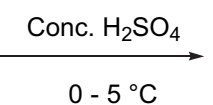<smiles>Cc1ccc2c(CBr)cc(=O)oc2c1</smiles><smiles>O=S(=O)(O)C1CC1</smiles><smiles>Cc1cc2oc(=O)cc(CBr)c2cc1S(=O)(=O)Cl</smiles>

2

Scheme 1. Synthesis of 4-azidomethyl-7-methylcoumarin-6-sulfonamide 5. 
singlet in the down field at $4.78 \mathrm{ppm}$. The $\mathrm{C} 3-\mathrm{H}$ of coumarin was observed as singlet at $6.47 \mathrm{ppm}$. The aromatic protons resonated in the region of $6.55-8.30 \mathrm{ppm}$. Further evidence for the formation of sulfonamides was obtained from the mass spectral data. The Mass spectrum of (5c) $\left(\mathrm{R}=-\mathrm{C}_{6} \mathrm{H}_{4}-\mathrm{CH}_{3}-p\right)$ shows $\mathrm{M}+\mathrm{H}$ peak at $m / z 385$.

\section{Biological screening}

\subsection{Anti-bacterial screening}

All the newly synthesized compounds were evaluated for their in vitro anti-bacterial activity against (i) Gram-positive bacteria: Streptococcus faecalis (MTCC 3382), Staphylococcus aureus (MTCC 3160), Bacillus subtilis (MTCC 297) and (ii) Gram-negative bacteria: Pseudomonas aeruginosa (MTCC 1034), Klebsiella pneumoniae (MTCC 3384) and Escherichia coli (MTCC 1089) by broth microdilution method [29]. The anti-bacterial data (Table 1 ) revealed that all the sulfonamides exhibited moderate activity a few of them highly active, but majority of them inactive against $E$. coli. Compounds with methoxy group $\mathbf{5 g}, \mathbf{5 h}$, $\mathbf{5 i}, \mathbf{5 j}, \mathbf{5 k}$ were highly active against $S$. faecalis with MIC of $1 \mu \mathrm{g} / \mathrm{mL}$. The $m$-methoxy compound $\mathbf{5 h}$ was found to equally effective against $P$. aeruginosa and K. pneumoniae with MIC of 1 and $2 \mu \mathrm{g} / \mathrm{mL}$, respectively. The 2-chloro compound $\mathbf{5 n}$ and 4-bromo compound $\mathbf{5 s}$ were found to be very active against $S$. faecalis, $P$. aeruginosa and $K$. pneumoniae. Many compounds exhibited MIC similar to ciprofloxacin against three bacterial species except $E$. coli. Highest activity was associated with mono-nitro, chloro, bromo and methoxy substituents.

\subsection{Anti-fungal screening}

All the sulfonamides were screened for their anti-fungal activity against Candida albicans, Aspergillus fumigatus, Aspergillus niger, Penicillium chrysogenum, Mucor fuscus and Fusarium oxysporum in DMSO by broth micro dilution method [30].

The screening data (Table 2) gives a spectrum of their activity and all of them were most active against $F$. oxysporum . The most active compounds against $C$. albicans were $\mathbf{5 c}, \mathbf{5 b}$ and $\mathbf{5 u}$ with 4 methyl, 3-methyl and 2-chloro-5-nitro substituents exhibiting MICs of 1,4 and $4 \mu \mathrm{g} / \mathrm{mL}$, respectively. The 2,6-dimethyl, 4methoxy, 2-chloro-5-nitro and naphthyl exhibited $2 \mu \mathrm{g} / \mathrm{mL}$ MIC against $A$. fumigatus. A broad spectrum of anti-fungal activity was observed in $\mathbf{5 b}, \mathbf{5 h}, \mathbf{5 i}, \mathbf{5 y}$ for $A$. niger. Other compounds those were effective at $2 \mu \mathrm{g} / \mathrm{mL}$ against $P$. chrysogenum were $\mathbf{5 a}, \mathbf{5 d}, \mathbf{5 1}, \mathbf{5 0}, \mathbf{5 r}$ and $\mathbf{5 x}$. The compounds $\mathbf{5 a}, \mathbf{5 d}, \mathbf{5 h}, \mathbf{5 l}$ and $\mathbf{5 x}$ showed significant activity against $M$. fuscus. It is interesting to note that all these compounds exhibited a better activity than fluconazole.

\section{Experimental}

\subsection{Chemistry}

The melting points were determined by open capillary method and are uncorrected. The IR spectra ( $\mathrm{KBr}$ disc) were recorded on a Nicolet-5700 FT-IR spectrophotometer. ${ }^{1} \mathrm{H}$ NMR spectra were recorded on Bruker $300 \mathrm{MHz}$ spectrometer using $\mathrm{CDCl}_{3}$ and DMSO$d_{6}$ as solvents and TMS as an internal standard. The chemical shifts are expressed in $\delta \mathrm{ppm}$. The mass spectra were recorded using Agilent-single Quartz LC-MS. The elemental analysis was carried out using Heraus CHN rapid analyzer. The purity of the compound was checked by T.L.C. All the chemicals purchased were of analytical grade, and were used without further purification unless otherwise stated.

\subsubsection{Synthesis of 4-bromomethyl-7-methylcoumarin (1)}

The required 4-bromomethyl-7-methylcoumarin [19] have been synthesized by the Pechmann cyclization of $m$-cresol with 4-bromoethylacetoacetate [31].

\subsubsection{Synthesis of 4-bromomethyl-7-methyl-coumarin-6-sulfonyl chloride (2)}

4-Bromomethyl-7-methylcoumarin 1 (2.53 g, $0.01 \mathrm{~mol}$ ) was taken in a two-necked R. B. flask with a dropping funnel and reflux

Table 1

Results of anti-bacterial activities of compounds $\mathbf{2 , 3}$ and $\mathbf{5 a}-\mathbf{y}$ MICs $(\mu \mathrm{g} / \mathrm{mL})$.

\begin{tabular}{|c|c|c|c|c|c|c|c|}
\hline \multirow[t]{2}{*}{ Compound } & \multirow[t]{2}{*}{$\mathrm{R}$} & \multicolumn{3}{|c|}{ Gram-positive } & \multicolumn{3}{|c|}{ Gram-negative } \\
\hline & & S. faecalis & S. aureus & B. subtilis & P. aeruginosa & K. pneumoniae & E. coli \\
\hline 2 & - & 16 & 16 & 16 & 125 & 125 & 125 \\
\hline 3 & - & 31.25 & 4 & 31.25 & 125 & 125 & 125 \\
\hline $5 a$ & $\mathrm{H}$ & 31.25 & 4 & 31.25 & 125 & 125 & 125 \\
\hline $5 \mathbf{b}$ & $3-\mathrm{CH}_{3}$ & 250 & 16 & 16 & 2 & 4 & $>500$ \\
\hline $5 c$ & $4-\mathrm{CH}_{3}$ & 16 & 16 & 8 & 125 & 1 & $>500$ \\
\hline $5 d$ & $3,4-\mathrm{CH}_{3}$ & 8 & 4 & 31.25 & 125 & 125 & 125 \\
\hline $5 e$ & $2,4-\mathrm{CH}_{3}$ & 62.5 & 16 & 125 & 4 & 62.5 & $>500$ \\
\hline $5 f$ & $2,6-\mathrm{CH}_{3}$ & $>500$ & 125 & $>500$ & 2 & 1 & $>500$ \\
\hline $5 g$ & $2-\mathrm{OCH}_{3}$ & 1 & 31.25 & $>500$ & 125 & 62.5 & 250 \\
\hline $5 h$ & $3-\mathrm{OCH}_{3}$ & 1 & 16 & $>500$ & 1 & 2 & $>500$ \\
\hline $5 i$ & $4-\mathrm{OCH}_{3}$ & 1 & 4 & $>500$ & 8 & 1 & 250 \\
\hline $5 \mathbf{j}$ & $4-\mathrm{OC}_{2} \mathrm{H}_{5}$ & 1 & 2 & $>500$ & 62.5 & 16 & 62.5 \\
\hline $5 k$ & $2-\mathrm{NO}_{2}$ & 1 & 1 & 62.5 & 4 & 16 & $>500$ \\
\hline 51 & $3-\mathrm{NO}_{2}$ & 8 & 4 & 8 & 125 & 125 & 125 \\
\hline $5 \mathrm{~m}$ & $4-\mathrm{NO}_{2}$ & 4 & 2 & 125 & 1 & 2 & 250 \\
\hline $5 n$ & $2-\mathrm{Cl}^{2}$ & 1 & 1 & 125 & 31.25 & 1 & $>500$ \\
\hline 50 & $3-\mathrm{Cl}$ & 8 & 1 & 16 & 1 & 4 & $>500$ \\
\hline $5 p$ & $4-\mathrm{Cl}$ & 4 & 8 & 16 & 1 & 1 & $>500$ \\
\hline $5 q$ & $2,4-\mathrm{Cl}$ & 1 & 31.25 & 250 & 2 & 2 & $>500$ \\
\hline $5 r$ & $3,4-\mathrm{Cl}$ & 16 & 4 & 2 & 8 & 1 & $>500$ \\
\hline $5 s$ & $4-\mathrm{Br}$ & 1 & 16 & $>500$ & 1 & 1 & $>500$ \\
\hline $5 t$ & $3-\mathrm{Cl}, 4-\mathrm{CH}_{3}$ & 8 & 8 & 4 & 1 & 8 & $>500$ \\
\hline $5 \mathbf{u}$ & $2-\mathrm{Cl}, 5-\mathrm{NO}_{2}$ & 2 & 2 & 125 & 2 & 62.5 & $>500$ \\
\hline $5 v$ & $2-\mathrm{NO}_{2}, 4-\mathrm{Cl}$ & 4 & 4 & 250 & 8 & 16 & $>500$ \\
\hline $5 w$ & $2-\mathrm{NO}_{2}, 4-\mathrm{CH}_{3}$ & 1 & 16 & 250 & 16 & 4 & 250 \\
\hline $5 x$ & $2-\mathrm{CH}_{3}, 4-\mathrm{NO}_{2}$ & 4 & 16 & 4 & 125 & 31.25 & 125 \\
\hline $5 y$ & Naphthylamine & 2 & 16 & $>500$ & 8 & 1 & $>500$ \\
\hline Ciprofloxacin & - & 1 & 1 & 1 & 1 & 1 & 1 \\
\hline
\end{tabular}


Table 2

Results of anti-fungal activities of compounds $\mathbf{2 , 3}$ and $\mathbf{5 a}-\mathbf{y}$ MICs $(\mu \mathrm{g} / \mathrm{mL})$.

\begin{tabular}{|c|c|c|c|c|c|c|c|}
\hline Compound & $\mathrm{R}$ & C. albicans & A. fumigatus & A. niger & P. chrysogenum & M. fuscus & F. oxysporum \\
\hline 2 & - & 8 & 4 & 4 & 1 & 1 & 1 \\
\hline 3 & - & 8 & 8 & 8 & 4 & 2 & 2 \\
\hline $5 a$ & $\mathrm{H}$ & 16 & 8 & 8 & 2 & 4 & 4 \\
\hline $5 \mathbf{b}$ & $3-\mathrm{CH}_{3}$ & 4 & 8 & 1 & 8 & 62.5 & 125 \\
\hline $5 c$ & $4-\mathrm{CH}_{3}$ & 1 & 16 & 4 & 16 & 62.5 & 1 \\
\hline $5 d$ & $3,4-\mathrm{CH}_{3}$ & 8 & 8 & 4 & 2 & 1 & 1 \\
\hline $5 e$ & $2,4-\mathrm{CH}_{3}$ & 8 & 16 & 4 & 31.25 & 16 & 1 \\
\hline $5 f$ & $2,6-\mathrm{CH}_{3}$ & 8 & 2 & 8 & 31.25 & 16 & 1 \\
\hline $5 g$ & $2-\mathrm{OCH}_{3}$ & 31.25 & 62.5 & 16 & $>500$ & 31.25 & 1 \\
\hline $5 h$ & $3-\mathrm{OCH}_{3}$ & 8 & 31.25 & 2 & 125 & 4 & 1 \\
\hline $5 \mathbf{i}$ & $4-\mathrm{OCH}_{3}$ & 8 & 2 & 2 & 250 & 250 & 1 \\
\hline $5 \mathbf{j}$ & $4-\mathrm{OC}_{2} \mathrm{H}_{5}$ & 16 & $>500$ & 250 & $>500$ & 62.5 & 1 \\
\hline $5 k$ & $2-\mathrm{NO}_{2}$ & 250 & 8 & 8 & 4 & 8 & 1 \\
\hline 51 & $3-\mathrm{NO}_{2}$ & 16 & 8 & 8 & 2 & 4 & 2 \\
\hline $5 \mathrm{~m}$ & $4-\mathrm{NO}_{2}$ & 62.5 & 250 & 62.5 & 62.5 & 8 & 1 \\
\hline $5 n$ & $2-\mathrm{Cl}$ & 62.5 & 31.25 & 62.5 & 31.25 & 16 & 4 \\
\hline 50 & $3-\mathrm{Cl}$ & $>500$ & 16 & 31.25 & 2 & $>500$ & 1 \\
\hline $5 p$ & $4-\mathrm{Cl}$ & 8 & 125 & 31.25 & 16 & 16 & 1 \\
\hline $5 q$ & $2,4-\mathrm{Cl}$ & 8 & 250 & 250 & 62.5 & 16 & 1 \\
\hline $5 r$ & $3,4-\mathrm{Cl}$ & 16 & 31.25 & 16 & 2 & 31.25 & 1 \\
\hline $5 s$ & $4-\mathrm{Br}$ & 125 & 500 & 500 & 16 & 250 & 1 \\
\hline $5 t$ & $3-\mathrm{Cl}, 4-\mathrm{CH}_{3}$ & 31.25 & 16 & 16 & 4 & 16 & 1 \\
\hline $5 \mathbf{u}$ & $2-\mathrm{Cl}, 5-\mathrm{NO}_{2}$ & 4 & 2 & 8 & 125 & 62.5 & 1 \\
\hline $5 v$ & $2-\mathrm{NO}_{2}, 4-\mathrm{Cl}$ & 16 & 500 & 62.5 & 125 & 8 & 1 \\
\hline $5 w$ & $2-\mathrm{NO}_{2}, 4-\mathrm{CH}_{3}$ & 16 & 125 & 31.25 & 31.25 & 62.5 & 1 \\
\hline $5 x$ & $2-\mathrm{CH}_{3}, 4-\mathrm{NO}_{2}$ & 16 & 8 & 4 & 2 & 2 & 2 \\
\hline $5 y$ & Naphthylamine & 8 & 2 & 1 & 16 & 31.25 & 1 \\
\hline Fluconazole & - & 8 & 8 & 8 & 8 & 8 & 8 \\
\hline
\end{tabular}

condenser containing a $\mathrm{CaCl}_{2}$ guard tube. Chlorosulfonic acid $(4 \mathrm{ml}$, $0.06 \mathrm{~mol}$ ) was added through a dropping funnel in small portion with occasional shaking. The reaction mixture was heated on a water bath for $1 \mathrm{~h}$ (monitored by TLC), cooled and poured on to crushed ice $(100 \mathrm{~g})$. The separated solid was filtered off, washed with water, dried and recrystallized from chloroform to obtain a colorless solid in 55\% yield. m.p. $170-171{ }^{\circ} \mathrm{C}$; IR $(\mathrm{KBr}) \mathrm{cm}^{-1} 1716$ $(\mathrm{C}=\mathrm{O}) ;{ }^{1} \mathrm{H} N M R\left(\mathrm{CDCl}_{3}, 300 \mathrm{MHz}, \mathrm{TMS}\right): \delta 2.89\left(\mathrm{~s}, 3 \mathrm{H}, \mathrm{C} 7-\mathrm{CH}_{3}\right), 4.52$ (s, 2H, C4-CH $), 6.63(\mathrm{~s}, 1 \mathrm{H}, \mathrm{C} 3-\mathrm{H}), 7.40(\mathrm{~s}, 1 \mathrm{H}, \mathrm{C} 8-\mathrm{H}), 8.45$ (s, 1H, C5H); LC-MS: $353(\mathrm{M}+2)$. Anal. Calcd for $\mathrm{C}_{11} \mathrm{H}_{8} \mathrm{BrClO}_{4} \mathrm{~S}$ (\%): Calcd. C, 37.58; H, 2.29. Found: C, 37.21; H, 2.02.

\subsubsection{Synthesis of 4-azidomethyl-7-methyl-coumarin-6-sulfonyl chloride (3)}

4-Bromomethyl-7-methyl-coumarin-6-sulfonyl chloride 2 (3.51 g, $0.01 \mathrm{~mol})$ was taken in acetone $(20 \mathrm{ml})$ in R. B. flask. To this, solution of sodium azide $(0.78 \mathrm{~g}, 0.012 \mathrm{~mol})$ in water $(3 \mathrm{ml})$ was added drop wise with stirring. The stirring was continued for $10 \mathrm{~h}$. Then, the reaction mixture was poured on to crushed ice (100 g). The separated solid was filtered and recrystallized from benzene to obtain a colorless solid in $65 \%$ yield. m.p. $120-121^{\circ} \mathrm{C}$; IR $(\mathrm{KBr}) \mathrm{cm}^{-1}$ $1728(\mathrm{C}=\mathrm{O}), 2135\left(\mathrm{~N}_{3}\right.$, azido); ${ }^{1} \mathrm{H}$ NMR $\left(\mathrm{CDCl}_{3}, 300 \mathrm{MHz}, \mathrm{TMS}\right)$ : $\delta 2.77\left(\mathrm{~s}, 3 \mathrm{H}, \mathrm{C} 7-\mathrm{CH}_{3}\right), 4.66\left(\mathrm{~s}, 2 \mathrm{H}, \mathrm{C} 4-\mathrm{CH}_{2}\right), 6.67(\mathrm{~s}, 1 \mathrm{H}, \mathrm{C} 3-\mathrm{H}), 7.39$ $(\mathrm{s}, 1 \mathrm{H}, \mathrm{C} 8-\mathrm{H}), 8.35(\mathrm{~s}, 1 \mathrm{H}, \mathrm{C} 5-\mathrm{H})$; Anal. Calcd for $\mathrm{C}_{11} \mathrm{H}_{8} \mathrm{ClN}_{3} \mathrm{O}_{4} \mathrm{~S}(\%)$ : Calcd. C, 42.11; H, 2.57; N, 13.39. Found: C, 42.01; H, 2.31; N, 13.11.

\subsubsection{Synthesis of 4-azidomethyl-7-methyl-coumarin-6-} sulfonamides (5): general procedure

To a mixture of 4-azidomethyl-7-methyl-coumarin-6-sulfonyl chloride 3 (3.13 g, $0.01 \mathrm{~mol})$ in dry benzene $(30 \mathrm{ml})$ the appropriate aromatic amines $4(0.015 \mathrm{~mol})$ were added and heated under reflux for $4 \mathrm{~h}$ (18-20 h for nitro amines) (monitored by TLC). The solvent was removed under reduced pressure. The residue obtained was filtered off and crystallized from benzene.

5.1.4.1. Synthesis of 4-azidomethyl-7-methyl-coumarin-6-sulfonic acid phenylamide (5a). Colorless solid; yield 70\%; m.p. $168{ }^{\circ} \mathrm{C}$; IR
$(\mathrm{KBr}) \mathrm{cm}^{-1} 1716(\mathrm{C}=\mathrm{O}), 2124\left(\mathrm{~N}_{3}\right), 3367(\mathrm{NH}) ;{ }^{1} \mathrm{H}$ NMR (DMSO, $300 \mathrm{MHz}, \mathrm{TMS}): \delta 2.67\left(\mathrm{~s}, 3 \mathrm{H}, \mathrm{C} 7-\mathrm{CH}_{3}\right), 5.12\left(\mathrm{~s}, 2 \mathrm{H}, \mathrm{C} 4-\mathrm{CH}_{2}\right), 6.37(\mathrm{~s}$, $1 \mathrm{H}, \mathrm{C} 3-\mathrm{H}), 6.63-8.39(\mathrm{~m}, 8 \mathrm{H}, \mathrm{Ar}-\mathrm{H}$ and $\mathrm{NH})$; Anal. Calcd for $\mathrm{C}_{17} \mathrm{H}_{14} \mathrm{~N}_{4} \mathrm{O}_{4} \mathrm{~S}$ (\%): Calcd. C, 55.13; H, 3.81; N, 15.13. Found: C, 55.02; $\mathrm{H}, 3.57 ; \mathrm{N}, 15.01$.

5.1.4.2. 4-Azidomethyl-7-methyl-coumarin-6-sulfonic acid m-tolylamide (5b). Yellow solid; yield 75\%; m.p. $110^{\circ} \mathrm{C}$; IR (KBr) cm ${ }^{-1} 1714$ $(\mathrm{C}=\mathrm{O}), 2120\left(\mathrm{~N}_{3}\right), 3345(\mathrm{NH}) ;{ }^{1} \mathrm{H}$ NMR (DMSO, $\left.300 \mathrm{MHz}, \mathrm{TMS}\right)$ : $\delta 2.66\left(\mathrm{~s}, 3 \mathrm{H}, \mathrm{C} 7-\mathrm{CH}_{3}\right), 3.51\left(\mathrm{~s}, 3 \mathrm{H}, 3-\mathrm{CH}_{3}\right.$ of phenyl), $4.59(\mathrm{~s}, 1 \mathrm{H}, \mathrm{NH}$, $\mathrm{D}_{2} \mathrm{O}$ exchangeable), $4.93\left(\mathrm{~s}, 2 \mathrm{H}, \mathrm{C} 4-\mathrm{CH}_{2}\right), 6.34(\mathrm{~s}, 1 \mathrm{H}, \mathrm{C} 3-\mathrm{H}), 6.55-$ $8.37(\mathrm{~m}, 6 \mathrm{H}, \mathrm{Ar}-\mathrm{H})$; LC-MS $385(\mathrm{M}+\mathrm{H})$. Anal. Calcd for $\mathrm{C}_{18} \mathrm{H}_{16} \mathrm{~N}_{4} \mathrm{O}_{4} \mathrm{~S}$ (\%): Calcd. C, 56.24; H, 4.20; N, 14.57. Found: C, 56.14; H, 4.13; N, 14.40 .

5.1.4.3. 4-Azidomethyl-7-methyl-coumarin-6-sulfonic acid p-tolylamide (5c). Brown solid; yield 72\%; m.p. $188^{\circ} \mathrm{C}$; IR (KBr) cm ${ }^{-1} 1710$ $(\mathrm{C}=\mathrm{O}), 2115\left(\mathrm{~N}_{3}\right), 3341(\mathrm{NH}) ;{ }^{1} \mathrm{H}$ NMR (DMSO, $\left.300 \mathrm{MHz}, \mathrm{TMS}\right)$ : $\delta 2.29$ (s, 3H, 4- $\mathrm{CH}_{3}$ of phenyl), $2.66\left(\mathrm{~s}, 3 \mathrm{H}, \mathrm{C} 7-\mathrm{CH}_{3}\right), 4.49(\mathrm{~s}, 1 \mathrm{H}, \mathrm{NH}$, $\mathrm{D}_{2} \mathrm{O}$ exchangeable), $4.78\left(\mathrm{~s}, 2 \mathrm{H}, \mathrm{C} 4-\mathrm{CH}_{2}\right), 6.47(\mathrm{~s}, 1 \mathrm{H}, \mathrm{C} 3-\mathrm{H}), 6.55-$ $8.30(\mathrm{~m}, 6 \mathrm{H}, \mathrm{Ar}-\mathrm{H})$; LC-MS $385(\mathrm{M}+\mathrm{H})$. Anal. Calcd for $\mathrm{C}_{18} \mathrm{H}_{16} \mathrm{~N}_{4} \mathrm{O}_{4} \mathrm{~S}$ (\%): Calcd. C, 56.24; H, 4.20; N, 14.57. Found: C, 56.04; H, 4.04; N, 14.39.

5.1.4.4. 4-Azidomethyl-7-methyl-coumarin-6-sulfonic acid (3,4dimethyl-phenyl)-amide (5d). Colorless solid; yield 70\%; m.p. $190{ }^{\circ} \mathrm{C}$; IR $(\mathrm{KBr}) \mathrm{cm}^{-1} 1712(\mathrm{C}=\mathrm{O}), 2115\left(\mathrm{~N}_{3}\right), 3352(\mathrm{NH}) ;{ }^{1} \mathrm{H}$ NMR (DMSO, $300 \mathrm{MHz}, \mathrm{TMS}): \delta 2.00\left(\mathrm{~s}, 3 \mathrm{H}, 4-\mathrm{CH}_{3}\right.$ of phenyl), $2.66(\mathrm{~s}, 3 \mathrm{H}$, $\left.\mathrm{C}^{-}-\mathrm{CH}_{3}\right), 3.90$ (s, 3H, 3- $\mathrm{CH}_{3}$ of phenyl), 5.00, (s, 2H, $\left.\mathrm{C}_{4}-\mathrm{CH}_{2}\right), 6.60$ (s, $1 \mathrm{H}, \mathrm{C} 3-\mathrm{H}), \quad 6.70-8.40(\mathrm{~m}, 5 \mathrm{H}, \mathrm{Ar}-\mathrm{H}), 10.0\left(\mathrm{~s}, 1 \mathrm{H}, \mathrm{NH}, \mathrm{D}_{2} \mathrm{O}\right.$ exchangeable). Anal. Calcd for $\mathrm{C}_{19} \mathrm{H}_{18} \mathrm{~N}_{4} \mathrm{O}_{4} \mathrm{~S}$ (\%): Calcd. C, 57.27; $\mathrm{H}$, 4.55; N, 14.06. Found: C, 57.03; H, 4.32; N, 13.92 .

5.1.4.5. 4-Azidomethyl-7-methyl-coumarin-6-sulfonic acid (2,4dimethyl-phenyl)-amide (5e). Brown solid; yield 69\%; m.p. $161{ }^{\circ} \mathrm{C}$; IR $(\mathrm{KBr}) \mathrm{cm}^{-1} 1730(\mathrm{C}=\mathrm{O}), 2134\left(\mathrm{~N}_{3}\right), 3440(\mathrm{NH}) ;{ }^{1} \mathrm{H}$ NMR (DMSO, $300 \mathrm{MHz}, \mathrm{TMS}): \delta 2.13\left(\mathrm{~s}, 6 \mathrm{H}, 2-\mathrm{CH}_{3}\right.$ and $4-\mathrm{CH}_{3}$ of phenyl), 2.69 (s, 
$\left.3 \mathrm{H}, \mathrm{C} 7-\mathrm{CH}_{3}\right), 5.16$ (s, 2H, C4-CH $), 6.35(\mathrm{~s}, 1 \mathrm{H}, \mathrm{C} 3-\mathrm{H}), 6.70-8.25(\mathrm{~m}$, $5 \mathrm{H}, \mathrm{Ar}-\mathrm{H}), 8.51\left(\mathrm{~s}, 1 \mathrm{H}, \mathrm{NH}, \mathrm{D}_{2} \mathrm{O}\right.$ exchangeable). Anal. Calcd for $\mathrm{C}_{19} \mathrm{H}_{18} \mathrm{~N}_{4} \mathrm{O}_{4} \mathrm{~S}$ (\%): Calcd. C, 57.27; H, 4.55; N, 14.06. Found: C, 57.12; $\mathrm{H}, 4.48 ; \mathrm{N}, 14.01$.

5.1.4.6. 4-Azidomethyl-7-methyl-coumarin-6-sulfonic acid (2,6dimethyl-phenyl)-amide (5f). Green solid; yield 65\%; m.p. $185^{\circ} \mathrm{C}$; IR $(\mathrm{KBr}) \mathrm{cm}^{-1} 1730(\mathrm{C}=\mathrm{O}), 2134\left(\mathrm{~N}_{3}\right), 3440(\mathrm{NH}) ;{ }^{1} \mathrm{H}$ NMR (DMSO, $300 \mathrm{MHz}, \mathrm{TMS}$ ): $\delta 2.13$ (s, 6H, 2- $\mathrm{CH}_{3}$ and $4-\mathrm{CH}_{3}$ of phenyl), 2.69 (s, $\left.3 \mathrm{H}, \mathrm{C} 7-\mathrm{CH}_{3}\right), 5.16\left(\mathrm{~s}, 2 \mathrm{H}, \mathrm{C} 4-\mathrm{CH}_{2}\right), 6.35(\mathrm{~s}, 1 \mathrm{H}, \mathrm{C} 3-\mathrm{H}), 6.70-8.25(\mathrm{~m}$, $5 \mathrm{H}, \mathrm{Ar}-\mathrm{H}), 8.51\left(\mathrm{~s}, 1 \mathrm{H}, \mathrm{NH}, \mathrm{D}_{2} \mathrm{O}\right.$ exchangeable). Anal. Calcd for $\mathrm{C}_{19} \mathrm{H}_{18} \mathrm{~N}_{4} \mathrm{O}_{4} \mathrm{~S}$ (\%): Calcd. C, 57.27; H, 4.55; N, 14.06. Found: C, 57.12; $\mathrm{H}, 4.48 ; \mathrm{N}, 13.97$.

5.1.4.7. 4-Azidomethyl-7-methyl-coumarin-6-sulfonic acid (2methoxy-phenyl)-amide (5g). Black solid; yield 75\%; m.p. $206{ }^{\circ} \mathrm{C}$; IR $(\mathrm{KBr}) \mathrm{cm}^{-1} 1739(\mathrm{C}=\mathrm{O}), 2143\left(\mathrm{~N}_{3}\right), 3433(\mathrm{NH}) ;{ }^{1} \mathrm{H}$ NMR (DMSO, $300 \mathrm{MHz}, \mathrm{TMS}): \delta 2.68\left(\mathrm{~s}, 3 \mathrm{H}, \mathrm{C} 7-\mathrm{CH}_{3}\right), 3.82\left(\mathrm{~s}, 3 \mathrm{H}, 2-\mathrm{OCH}_{3}\right.$ of phenyl), $4.66\left(\mathrm{~s}, 2 \mathrm{H}, \mathrm{C} 4-\mathrm{CH}_{2}\right), 6.27(\mathrm{~s}, 1 \mathrm{H}, \mathrm{C} 3-\mathrm{H}), 6.47-8.28(\mathrm{~m}, 6 \mathrm{H}$, Ar- $\mathrm{H}), 8.41\left(\mathrm{~s}, 1 \mathrm{H}, \mathrm{NH}, \mathrm{D}_{2} \mathrm{O}\right.$ exchangeable). Anal. Calcd for $\mathrm{C}_{18} \mathrm{H}_{16} \mathrm{~N}_{4} \mathrm{O}_{5} \mathrm{~S}$ (\%): Calcd. C, 53.99; H, 4.03; N, 13.99. Found: C, 53.90; $\mathrm{H}, 3.92 ; \mathrm{N}, 13.94$.

5.1.4.8. 4-Azidomethyl-7-methyl-coumarin-6-sulfonic acid (3methoxy-phenyl)-amide (5h). Green solid; yield 77\%; m.p. $119{ }^{\circ} \mathrm{C}$; IR $(\mathrm{KBr}) \mathrm{cm}^{-1} 1725(\mathrm{C}=\mathrm{O}), 2123\left(\mathrm{~N}_{3}\right), 3372(\mathrm{NH}) ;{ }^{1} \mathrm{H}$ NMR (DMSO, $300 \mathrm{MHz}, \mathrm{TMS}): \delta 2.66\left(\mathrm{~s}, 3 \mathrm{H}, \mathrm{C} 7-\mathrm{CH}_{3}\right), 3.58\left(\mathrm{~s}, 3 \mathrm{H}, 3-\mathrm{OCH}_{3}\right.$ of phenyl), 4.55 (s, $1 \mathrm{H}, \mathrm{NH}, \mathrm{D}_{2} \mathrm{O}$ exchangeable), $4.93\left(\mathrm{~s}, 2 \mathrm{H}, \mathrm{C} 4-\mathrm{CH}_{2}\right)$, 6.38 (s, 1H, C3-H), 7.65 (s, 1H, C8-H), 6.61-8.21 (m, 4H, Ar-H), 8.38 (s, 1H, C5-H); LC-MS $401(\mathrm{M}+\mathrm{H})$. Anal. Calcd for $\mathrm{C}_{18} \mathrm{H}_{16} \mathrm{~N}_{4} \mathrm{O}_{5} \mathrm{~S}(\%)$ : Calcd. C, 53.99; H, 4.03; N, 13.99. Found: C, 53.90; H, 3.95; N, 13.92.

5.1.4.9. 4-Azidomethyl-7-methyl-coumarin-6-sulfonic acid (4methoxy-phenyl)-amide (5i). Green solid; yield 74\%; m.p. $200{ }^{\circ} \mathrm{C}$; IR $(\mathrm{KBr}) \mathrm{cm}^{-1} 1714(\mathrm{C}=\mathrm{O}), 2123\left(\mathrm{~N}_{3}\right), 3356(\mathrm{NH}) ;{ }^{1} \mathrm{H}$ NMR (DMSO, $300 \mathrm{MHz}, \mathrm{TMS}): \delta 2.67\left(\mathrm{~s}, 3 \mathrm{H}, \mathrm{C} 7-\mathrm{CH}_{3}\right), 3.39\left(\mathrm{~s}, 3 \mathrm{H}, 4-\mathrm{OCH}_{3}\right.$ of phenyl), 4.83 (s, 2H, C4- $\left.\mathrm{CH}_{2}\right), 6.58(\mathrm{~s}, 1 \mathrm{H}, \mathrm{C} 3-\mathrm{H}), 7.05-7.48(\mathrm{~m}, 5 \mathrm{H}$, $\mathrm{Ar}-\mathrm{H}), 7.57$ (s, 1H, NH, $\mathrm{D}_{2} \mathrm{O}$ exchangeable), 8.14 (s, 1H, C5-H); LC-MS $401(\mathrm{M}+\mathrm{H})$. Anal. Calcd for $\mathrm{C}_{18} \mathrm{H}_{16} \mathrm{~N}_{4} \mathrm{O}_{5} \mathrm{~S}$ (\%): Calcd. C, 53.99; $\mathrm{H}$, 4.03; N, 13.99. Found: C, 53.94; H, 3.99; N, 13.94 .

5.1.4.10. 4-Azidomethyl-7-methyl-coumarin-6-sulfonic acid (4ethoxy-phenyl)-amide (5j). Green solid; yield 70\%; m.p. $191{ }^{\circ} \mathrm{C}$; IR $(\mathrm{KBr}) \mathrm{cm}^{-1} 1705(\mathrm{C}=\mathrm{O}), 2115\left(\mathrm{~N}_{3}\right), 3325(\mathrm{NH}) ;{ }^{1} \mathrm{H}$ NMR (DMSO, $300 \mathrm{MHz}, \mathrm{TMS}): \delta 1.31\left(\mathrm{t}, 3 \mathrm{H}, \mathrm{CH}_{3}\right.$ of $\left.\mathrm{C}_{2} \mathrm{H}_{5}\right), 2.64\left(\mathrm{~s}, 3 \mathrm{H}, \mathrm{C} 7-\mathrm{CH}_{3}\right), 3.46$ (q, $2 \mathrm{H}, \mathrm{CH}_{2}$ of $\left.\mathrm{C}_{2} \mathrm{H}_{5}\right), 4.51\left(\mathrm{~s}, 2 \mathrm{H}, \mathrm{C}_{-}-\mathrm{CH}_{2}\right), 6.37(\mathrm{~s}, 1 \mathrm{H}, \mathrm{C} 3-\mathrm{H}), 6.51-$ $7.34(\mathrm{~m}, 5 \mathrm{H}, \mathrm{Ar}-\mathrm{H}), 7.57$ (s, $1 \mathrm{H}, \mathrm{NH}, \mathrm{D}_{2} \mathrm{O}$ exchangeable), $8.35(\mathrm{~s}, 1 \mathrm{H}$, C5-H); Anal. Calcd for $\mathrm{C}_{19} \mathrm{H}_{18} \mathrm{~N}_{4} \mathrm{O}_{5} \mathrm{~S}$ (\%): Calcd. C, 55.06; H, 4.38; N, 13.52. Found: C, 55.00; H, 4.29; N, 13.48 .

5.1.4.11. 4-Azidomethyl-7-methyl-coumarin-6-sulfonic acid (2-nitrophenyl)-amide (5k). Yellow solid; yield 64\%; m.p. $101{ }^{\circ} \mathrm{C}$; IR ( $\left.\mathrm{KBr}\right)$ $\mathrm{cm}^{-1} 1725(\mathrm{C}=\mathrm{O}), 2122\left(\mathrm{~N}_{3}\right), 3351(\mathrm{NH}) ;{ }^{1} \mathrm{H}$ NMR (DMSO, $300 \mathrm{MHz}$, TMS): $\delta 2.68\left(\mathrm{~s}, 3 \mathrm{H}, \mathrm{C} 7-\mathrm{CH}_{3}\right), 4.98\left(\mathrm{~s}, 2 \mathrm{H}, \mathrm{C} 4-\mathrm{CH}_{2}\right), 6.63(\mathrm{~s}, 1 \mathrm{H}, \mathrm{C} 3-\mathrm{H})$, 6.91-8.09 (m, 5H, Ar-H), $8.24(\mathrm{~s}, 1 \mathrm{H}, \mathrm{C} 5-\mathrm{H}), 8.40\left(\mathrm{~s}, 1 \mathrm{H}, \mathrm{NH}, \mathrm{D}_{2} \mathrm{O}\right.$ exchangeable). Anal. Calcd for $\mathrm{C}_{17} \mathrm{H}_{13} \mathrm{~N}_{5} \mathrm{O}_{6} \mathrm{~S}$ (\%): Calcd. C, 49.16; $\mathrm{H}$, 3.15; N, 16.86. Found: C, 49.09; H, 3.08; N, 16.73.

5.1.4.12. 4-Azidomethyl-7-methyl-coumarin-6-sulfonic acid (3-nitrophenyl)-amide (5l). Yellow solid; yield 62\%; m.p. $101{ }^{\circ} \mathrm{C}$; IR ( $\left.\mathrm{KBr}\right)$ $\mathrm{cm}^{-1} 1727(\mathrm{C}=\mathrm{O}), 2099\left(\mathrm{~N}_{3}\right), 3344(\mathrm{NH}) ;{ }^{1} \mathrm{H}$ NMR (DMSO, $300 \mathrm{MHz}$, TMS): $\delta 2.50\left(\mathrm{~s}, 3 \mathrm{H}, \mathrm{C} 7-\mathrm{CH}_{3}\right), 4.80\left(\mathrm{~s}, 2 \mathrm{H}, \mathrm{C} 4-\mathrm{CH}_{2}\right), 6.20(\mathrm{~s}, 1 \mathrm{H}, \mathrm{C} 3-\mathrm{H})$, 6.30-9.17 (m, 7H, Ar-H and $\mathrm{NH})$. Anal. Calcd for $\mathrm{C}_{17} \mathrm{H}_{13} \mathrm{~N}_{5} \mathrm{O}_{6} \mathrm{~S}$ (\%): Calcd. C, 49.16; H, 3.15; N, 16.86. Found: C, 49.02; H, 3.01; N, 16.81 .
5.1.4.13. 4-Azidomethyl-7-methyl-coumarin-6-sulfonic acid (4-nitrophenyl)-amide (5m). Yellow solid; yield 61\%; m.p. $165{ }^{\circ} \mathrm{C}$; IR ( $\left.\mathrm{KBr}\right)$ $\mathrm{cm}^{-1} 1726(\mathrm{C}=\mathrm{O}), 2123\left(\mathrm{~N}_{3}\right), 3431(\mathrm{NH}) ;{ }^{1} \mathrm{H}$ NMR (DMSO, $300 \mathrm{MHz}$, TMS): $\delta 2.68\left(\mathrm{~s}, 3 \mathrm{H}, \mathrm{C} 7-\mathrm{CH}_{3}\right), 4.98\left(\mathrm{~s}, 2 \mathrm{H}, \mathrm{C}_{4}-\mathrm{CH}_{2}\right), 6.58(\mathrm{~s}, 1 \mathrm{H}, \mathrm{C} 3-\mathrm{H})$, 6.81-7.95 (m, 5H, Ar-H), $8.24(\mathrm{~s}, 1 \mathrm{H}, \mathrm{C} 5-\mathrm{H}), 8.40\left(\mathrm{~s}, 1 \mathrm{H}, \mathrm{NH}, \mathrm{D}_{2} \mathrm{O}\right.$ exchangeable). Anal. Calcd for $\mathrm{C}_{17} \mathrm{H}_{13} \mathrm{~N}_{5} \mathrm{O}_{6} \mathrm{~S}$ (\%): Calcd. C, 49.16; $\mathrm{H}$, 3.15; N, 16.86. Found: C, 49.10; H, 3.08; N, 16.78.

5.1.4.14. 4-Azidomethyl-7-methyl-coumarin-6-sulfonic acid (2chloro-phenyl)-amide (5n). Brown solid; yield 65\%; m.p. $184{ }^{\circ} \mathrm{C}$; IR $(\mathrm{KBr}) \mathrm{cm}^{-1} 1728(\mathrm{C}=0), 2125\left(\mathrm{~N}_{3}\right), 3409(\mathrm{NH}) ;{ }^{1} \mathrm{H}$ NMR (DMSO, $300 \mathrm{MHz}, \mathrm{TMS}): \delta 2.67$ (s, 3H, C7-CH $), 4.97\left(\mathrm{~s}, 2 \mathrm{H}, \mathrm{C}_{-}-\mathrm{CH}_{2}\right), 6.63(\mathrm{~s}$, 1H, C3-H), 6.80-7.68 (m, 5H, Ar-H), 8.23 (s, 1H, C5-H), 8.39 (s, 1H, $\mathrm{NH}, \mathrm{D}_{2} \mathrm{O}$ exchangeable). Anal. Calcd for $\mathrm{C}_{17} \mathrm{H}_{13} \mathrm{ClN}_{4} \mathrm{O}_{4} \mathrm{~S}$ (\%): Calcd. C, 50.44; H, 3.24; N, 13.84. Found: C, 50.32; H, 3.16; N, 13.78.

5.1.4.15. 4-Azidomethyl-7-methyl-coumarin-6-sulfonic acid (3chloro-phenyl)-amide (5o). Grey solid; yield 61\%; m.p. $196{ }^{\circ} \mathrm{C}$; IR $(\mathrm{KBr}) \mathrm{cm}^{-1} 1719(\mathrm{C}=\mathrm{O}), 2128\left(\mathrm{~N}_{3}\right), 3395(\mathrm{NH}) ;{ }^{1} \mathrm{H}$ NMR (DMSO, $300 \mathrm{MHz}, \mathrm{TMS}): \delta 2.68\left(\mathrm{~s}, 3 \mathrm{H}, \mathrm{C} 7-\mathrm{CH}_{3}\right), 4.98\left(\mathrm{~s}, 2 \mathrm{H}, \mathrm{C}_{-}-\mathrm{CH}_{2}\right), 6.34(\mathrm{~s}$, 1H, C3-H), 6.58-7.69 (m, 5H, Ar-H), $8.24(\mathrm{~s}, 1 \mathrm{H}, \mathrm{C} 5-\mathrm{H}), 8.38(\mathrm{~s}, 1 \mathrm{H}$, $\mathrm{NH}, \mathrm{D}_{2} \mathrm{O}$ exchangeable); LC-MS $405(\mathrm{M}+\mathrm{H})$. Anal. Calcd for $\mathrm{C}_{17} \mathrm{H}_{13} \mathrm{ClN}_{4} \mathrm{O}_{4} \mathrm{~S}$ (\%): Calcd. C, 50.44; H, 3.24; N, 13.84. Found: C, 50.38; H, 3.20; N, 13.80 .

5.1.4.16. 4-Azidomethyl-7-methyl-coumarin-6-sulfonic acid (4chloro-phenyl)-amide (5p). Colorless solid; yield 63\%; m.p. $140{ }^{\circ} \mathrm{C}$; IR (KBr) cm ${ }^{-1} 1725(\mathrm{C}=\mathrm{O}), 2122\left(\mathrm{~N}_{3}\right), 3395(\mathrm{NH}) ;{ }^{1} \mathrm{H}$ NMR (DMSO, $300 \mathrm{MHz}, \mathrm{TMS}): \delta 2.66\left(\mathrm{~s}, 3 \mathrm{H}, \mathrm{C} 7-\mathrm{CH}_{3}\right), 4.93\left(\mathrm{~s}, 2 \mathrm{H}, \mathrm{C}_{4}-\mathrm{CH}_{2}\right), 6.62(\mathrm{~s}$, $1 \mathrm{H}, \mathrm{C} 3-\mathrm{H}), 7.10-7.65(\mathrm{~m}, 5 \mathrm{H}, \mathrm{Ar}-\mathrm{H}), 8.22\left(\mathrm{~s}, 1 \mathrm{H}, \mathrm{C}_{5}-\mathrm{H}\right), 8.36(\mathrm{~s}, 1 \mathrm{H}$, $\mathrm{NH}, \mathrm{D}_{2} \mathrm{O}$ exchangeable). Anal. Calcd for $\mathrm{C}_{17} \mathrm{H}_{13} \mathrm{ClN}_{4} \mathrm{O}_{4} \mathrm{~S}$ (\%): Calcd. C, 50.44; H, 3.24; N, 13.84. Found: C, 50.39; H, 3.20; N, 13.76.

5.1.4.17. 4-Azidomethyl-7-methyl-coumarin-6-sulfonic acid (2,4dichloro-phenyl)-amide (5q). Black solid; yield 67\%; m.p. $165{ }^{\circ} \mathrm{C}$; IR $(\mathrm{KBr}) \mathrm{cm}^{-1} 1726(\mathrm{C}=0), 2121\left(\mathrm{~N}_{3}\right), 3429(\mathrm{NH}) ;{ }^{1} \mathrm{H}$ NMR (DMSO, $300 \mathrm{MHz}, \mathrm{TMS}): \delta 2.68\left(\mathrm{~s}, 3 \mathrm{H}, \mathrm{C} 7-\mathrm{CH}_{3}\right), 4.98\left(\mathrm{~s}, 2 \mathrm{H}, \mathrm{C}_{-}-\mathrm{CH}_{2}\right), 6.63(\mathrm{~s}$, $1 \mathrm{H}, \mathrm{C} 3-\mathrm{H}), 6.81-7.71$ (m, 4H, Ar-H), $8.24\left(\mathrm{~s}, 1 \mathrm{H}, \mathrm{C}_{5}-\mathrm{H}\right), 8.40(\mathrm{~s}, 1 \mathrm{H}$, $\mathrm{NH}, \mathrm{D}_{2} \mathrm{O}$ exchangeable). Anal. Calcd for $\mathrm{C}_{17} \mathrm{H}_{12} \mathrm{Cl}_{2} \mathrm{~N}_{4} \mathrm{O}_{4} \mathrm{~S}$ (\%): Calcd. C, 46.48; H, 2.75; N, 12.75. Found: C, 46.41; H, 2.71; N, 12.70.

5.1.4.18. 4-Azidomethyl-7-methyl-coumarin-6-sulfonic acid (3,4dichloro-phenyl)-amide (5r). Grey solid; yield 62\%; m.p. $196{ }^{\circ} \mathrm{C}$; IR $(\mathrm{KBr}) \mathrm{cm}^{-1} 1726(\mathrm{C}=\mathrm{O}), 2123\left(\mathrm{~N}_{3}\right), 3374(\mathrm{NH}) ;{ }^{1} \mathrm{H}$ NMR (DMSO, $300 \mathrm{MHz}, \mathrm{TMS}): \delta 2.68$ (s, 3H, C7-CH $), 4.98\left(\mathrm{~s}, 2 \mathrm{H}, \mathrm{C}_{-}-\mathrm{CH}_{2}\right), 6.63(\mathrm{~s}$, $1 \mathrm{H}, \mathrm{C} 3-\mathrm{H}), 6.81-7.68(\mathrm{~m}, 4 \mathrm{H}, \mathrm{Ar}-\mathrm{H}), 8.23\left(\mathrm{~s}, 1 \mathrm{H}, \mathrm{C}_{5}-\mathrm{H}\right), 8.37(\mathrm{~s}, 1 \mathrm{H}$, $\mathrm{NH}, \mathrm{D}_{2} \mathrm{O}$ exchangeable). Anal. Calcd for $\mathrm{C}_{17} \mathrm{H}_{12} \mathrm{Cl}_{2} \mathrm{~N}_{4} \mathrm{O}_{4} \mathrm{~S}$ (\%): Calcd. C, 46.48; H, 2.75; N, 12.75. Found: C, 46.45; H, 2.69; N, 12.67.

5.1.4.19. 4-Azidomethyl-7-methyl-coumarin-6-sulfonic acid (4bromo-phenyl)-amide (5s). Brown solid; yield 58\%; m.p. $175^{\circ} \mathrm{C}$; IR $(\mathrm{KBr}) \mathrm{cm}^{-1} 1709(\mathrm{C}=\mathrm{O}), 2112\left(\mathrm{~N}_{3}\right), 3354(\mathrm{NH}) ;{ }^{1} \mathrm{H}$ NMR (DMSO, $300 \mathrm{MHz}, \mathrm{TMS}$ ): $\delta 2.65$ (s, 3H, C7-CH $), 4.91\left(\mathrm{~s}, 2 \mathrm{H}, \mathrm{C}_{4}-\mathrm{CH}_{2}\right), 6.38(\mathrm{~s}$, $1 \mathrm{H}, \mathrm{C} 3-\mathrm{H}), 6.61-8.38(\mathrm{~m}, 7 \mathrm{H}, \mathrm{Ar}-\mathrm{H}$ and $\mathrm{NH})$. Anal. Calcd for $\mathrm{C}_{17} \mathrm{H}_{13} \mathrm{BrN}_{4} \mathrm{O}_{4} \mathrm{~S}$ (\%): Calcd. C, 45.45; H, 2.92; N, 12.47. Found: C, 45.37; H, 2.88; N, 12.37 .

5.1.4.20. 4-Azidomethyl-7-methyl-coumarin-6-sulfonic acid (3chloro-4-methyl-phenyl)-amide (5t). Yellow solid; yield 60\%; m.p. $154{ }^{\circ} \mathrm{C}$; IR (KBr) cm ${ }^{-1} 1726(\mathrm{C}=\mathrm{O}), 2135\left(\mathrm{~N}_{3}\right), 3374(\mathrm{NH}) ;{ }^{1} \mathrm{H}$ NMR (DMSO, $300 \mathrm{MHz}, \mathrm{TMS}): \delta 2.15\left(\mathrm{~s}, 3 \mathrm{H}, 4-\mathrm{CH}_{3}\right.$ of phenyl), $2.68(\mathrm{~s}, 3 \mathrm{H}$, $\left.\mathrm{C}_{-}-\mathrm{CH}_{3}\right), 4.98\left(\mathrm{~s}, 2 \mathrm{H}, \mathrm{C} 4-\mathrm{CH}_{2}\right), 6.34(\mathrm{~s}, 1 \mathrm{H}, \mathrm{C} 3-\mathrm{H}), 6.56-7.75(\mathrm{~m}, 4 \mathrm{H}$, $\mathrm{Ar}-\mathrm{H}), 8.24$ (s, 1H, C5-H), 8.39 (s, 1H, NH, $\mathrm{D}_{2} \mathrm{O}$ exchangeable). Anal. Calcd for $\mathrm{C}_{18} \mathrm{H}_{15} \mathrm{ClN}_{4} \mathrm{O}_{4} \mathrm{~S}$ (\%): Calcd. C, 51.62; H, 3.61; N, 13.38 . Found: C, 51.58; H, 3.55; N, 13.29. 
5.1.4.21. 4-Azidomethyl-7-methyl-coumarin-6-sulfonic acid (2chloro-5-nitro-phenyl)-amide (5u). Yellow solid; yield 58\%; m.p. $153{ }^{\circ} \mathrm{C}$; IR ( KBr) cm ${ }^{-1} 1729(\mathrm{C}=\mathrm{O}), 2122\left(\mathrm{~N}_{3}\right), 3429(\mathrm{NH}) ;{ }^{1} \mathrm{H}$ NMR (DMSO, $300 \mathrm{MHz}, \mathrm{TMS}): \delta 2.68\left(\mathrm{~s}, 3 \mathrm{H}, \mathrm{C} 7-\mathrm{CH}_{3}\right), 4.99\left(\mathrm{~s}, 2 \mathrm{H}, \mathrm{C} 4-\mathrm{CH}_{2}\right.$ ), $6.06(\mathrm{~s}, 1 \mathrm{H}, \mathrm{C} 3-\mathrm{H}), 6.65-7.62(\mathrm{~m}, 4 \mathrm{H}, \mathrm{Ar}-\mathrm{H}), 8.23(\mathrm{~s}, 1 \mathrm{H}, \mathrm{C} 5-\mathrm{H}), 8.41$ (s, $1 \mathrm{H}, \mathrm{NH}, \mathrm{D}_{2} \mathrm{O}$ exchangeable). Anal. Calcd for $\mathrm{C}_{17} \mathrm{H}_{12} \mathrm{ClN}_{5} \mathrm{O}_{6} \mathrm{~S}$ (\%): Calcd. C, 45.39; H, 2.69; N, 15.57. Found: C, 45.32; H, 2.61; N, 15.50 .

5.1.4.22. 4-Azidomethyl-7-methyl-coumarin-6-sulfonic acid (4chloro-2-nitro-phenyl)-amide (5v). Yellow solid; yield 56\%; m.p. $189{ }^{\circ} \mathrm{C}$; IR ( $\left.\mathrm{KBr}\right) \mathrm{cm}^{-1} 1726(\mathrm{C}=\mathrm{O}), 2122\left(\mathrm{~N}_{3}\right), 3430(\mathrm{NH}) ;{ }^{1} \mathrm{H}$ NMR (DMSO, $300 \mathrm{MHz}, \mathrm{TMS}): \delta 2.68\left(\mathrm{~s}, 3 \mathrm{H}, \mathrm{C} 7-\mathrm{CH}_{3}\right), 4.98\left(\mathrm{~s}, 2 \mathrm{H}, \mathrm{C} 4-\mathrm{CH}_{2}\right.$ ), $6.63(\mathrm{~s}, 1 \mathrm{H}, \mathrm{C} 3-\mathrm{H}), 6.81-7.69(\mathrm{~m}, 4 \mathrm{H}, \mathrm{Ar}-\mathrm{H}), 7.93\left(\mathrm{~s}, 1 \mathrm{H}, \mathrm{NH}, \mathrm{D}_{2} \mathrm{O}\right.$ exchangeable), $8.23(\mathrm{~s}, 1 \mathrm{H}, \mathrm{C} 5-\mathrm{H})$. Anal. Calcd for $\mathrm{C}_{17} \mathrm{H}_{12} \mathrm{ClN}_{5} \mathrm{O}_{6} \mathrm{~S}(\%)$ : Calcd. C, 45.39; H, 2.69; N, 15.57. Found: C, 45.35; H, 2.64; N, 15.54.

5.1.4.23. 4-Azidomethyl-7-methyl-coumarin-6-sulfonic acid (4methyl-2-nitro-phenyl)-amide (5w). Yellow solid; yield 59\%; m.p. $179{ }^{\circ} \mathrm{C}$; IR (KBr) cm ${ }^{-1} 1725(\mathrm{C}=\mathrm{O}), 2122\left(\mathrm{~N}_{3}\right), 3347(\mathrm{NH}) ;{ }^{1} \mathrm{H}$ NMR (DMSO, $300 \mathrm{MHz}, \mathrm{TMS}): \delta 2.18\left(\mathrm{~s}, 3 \mathrm{H}, 4-\mathrm{CH}_{3}\right.$ of phenyl), $2.67(\mathrm{~s}, 3 \mathrm{H}$, $\left.\mathrm{C}_{-}-\mathrm{CH}_{3}\right), 4.97$ (s, 2H, C4-CH $), 6.63(\mathrm{~s}, 1 \mathrm{H}, \mathrm{C} 3-\mathrm{H}), 6.80-7.68(\mathrm{~m}, 4 \mathrm{H}$, Ar-H), $7.74\left(\mathrm{~s}, 1 \mathrm{H}, \mathrm{NH}, \mathrm{D}_{2} \mathrm{O}\right.$ exchangeable), 8.23 (s, 1H, C5-H). Anal. Calcd for $\mathrm{C}_{18} \mathrm{H}_{15} \mathrm{~N}_{5} \mathrm{O}_{6} \mathrm{~S}$ (\%): Calcd. C, 50.35; H, 3.52; N, 16.31. Found: C, 50.29; H, 3.41; N, 16.27.

5.1.4.24. 4-Azidomethyl-7-methyl-coumarin-6-sulfonic acid (2methyl-4-nitro-phenyl)-amide (5x). Yellow solid; yield 60\%; m.p. $110{ }^{\circ} \mathrm{C}$; IR (KBr) cm ${ }^{-1} 1720(\mathrm{C}=\mathrm{O}), 2121\left(\mathrm{~N}_{3}\right), 3360(\mathrm{NH}) ;{ }^{1} \mathrm{H}$ NMR (DMSO, $300 \mathrm{MHz}, \mathrm{TMS}): \delta 2.08\left(\mathrm{~s}, 3 \mathrm{H}, 2-\mathrm{CH}_{3}\right.$ of phenyl), $2.65(\mathrm{~s}, 3 \mathrm{H}$, $\left.\mathrm{C}_{-}-\mathrm{CH}_{3}\right), 5.39\left(\mathrm{~s}, 2 \mathrm{H}, \mathrm{C} 4-\mathrm{CH}_{2}\right), 6.57(\mathrm{~s}, 1 \mathrm{H}, \mathrm{C} 3-\mathrm{H}), 6.72-8.27(\mathrm{~m}, 6 \mathrm{H}$, Ar-H and NH). Anal. Calcd for $\mathrm{C}_{18} \mathrm{H}_{15} \mathrm{~N}_{5} \mathrm{O}_{6} \mathrm{~S}$ (\%): Calcd. C, 50.35; $\mathrm{H}$, 3.52; N, 16.31. Found: C, 50.11; H, 3.29; N, 16.12.

5.1.4.25. 4-Azidomethyl-7-methyl-coumarin-6-sulfonic acid naphthalen-1-ylamide (5y). Grey solid; yield 64\%; m.p. $199{ }^{\circ} \mathrm{C}$; IR ( $\left.\mathrm{KBr}\right)$ $\mathrm{cm}^{-1} 1729(\mathrm{C}=\mathrm{O}), 2128\left(\mathrm{~N}_{3}\right), 3410(\mathrm{NH}) ;{ }^{1} \mathrm{H}$ NMR (DMSO, $300 \mathrm{MHz}$, TMS): $\delta 2.69$ (s, 3H, C7-CH 3 ), 4.98 (s, 2H, C4-CH $), 6.39-8.05(\mathrm{~m}, 9 \mathrm{H}$, Ar-H), 8.24 (s, 1H, C5-H), 8.55 (s, 1H, NH, $\mathrm{D}_{2} \mathrm{O}$ exchangeable). Anal. Calcd for $\mathrm{C}_{21} \mathrm{H}_{16} \mathrm{~N}_{4} \mathrm{O}_{4} \mathrm{~S}$ (\%): Calcd. C, 59.99; $\mathrm{H}, 3.84 ; \mathrm{N}, 13.33$. Found: C, 59.85; H, 3.78; N, 13.27.

\subsection{Anti-bacterial screening}

Standard strains were procured from the microbial type culture collection and Gene Bank (MTCC), Institute of Microbial Technology, Chandigarh, India. The anti-bacterial activity of the synthesized compounds $\mathbf{2 , 3}, \mathbf{5 a}-\mathbf{y}$ were performed in vitro against (i) Gram-positive bacteria: S. faecalis (MTCC 3382), S. aureus (MTCC 3160), B. subtilis (MTCC 297) and (ii) Gram-negative bacteria: $P$. aeruginosa (MTCC 1034), K. pneumoniae (MTCC 3384) and E. coli (MTCC 1089) by broth micro dilution method [29].

The MIC determination of the tested compounds was investigated in comparison with Ciprofloxacin. Double dilutions of the test compounds and reference drugs were prepared in Muller-Hinton agar. $10 \mathrm{mg}$ of each test compounds were dissolved in $1 \mathrm{ml}$ of dimethyl sulfoxide (DMSO) separately to prepare stock solution. Further, progressive dilutions with melted Mueller-Hinton agar were performed to obtain the required concentrations of 500, 250, $125,62.5,31.25,16,8,4,2,1 \mu \mathrm{g} / \mathrm{ml}$. The Petri dishes were inoculated with $1-5 \times 10^{4}$ colony forming units $\left(\mathrm{cfu} / \mathrm{ml}\right.$ ) and incubated at $37^{\circ} \mathrm{C}$ for 18 hours. The minimum inhibitory concentration (MIC) was the lowest concentration of the tested compounds that yield no visible growth on the plate was recorded in Table 1 . To ensure that the solvent had no effect on the bacterial growth, a control was performed with the test medium supplemented with DMSO at the same dilutions as used in the experiments.

\subsection{Anti-fungal screening}

The anti-fungal activity was performed against the following standard fungal strains: C. albicans, A. fumigatus, A. niger, P. chrysogenum, M. fuscus and F. oxysporum.

The MIC determination of the tested compounds was investigated in comparison with Fluconazole by broth micro dilution method [30]. Double dilutions of the test compounds and reference drugs were prepared in Sabouraud's dextrose broth. $10 \mathrm{mg}$ of each test compounds were dissolved in $1 \mathrm{ml}$ of dimethyl sulfoxide (DMSO) separately to prepare stock solution. Further progressive dilutions with Sabouraud's dextrose broth were performed to obtain the required concentrations of $500,250,125,62.5,31.25,16$, $8,4,2,1 \mu \mathrm{g} / \mathrm{ml}$. The Petri dishes were inoculated with $1-5 \times 10^{4}$ colony forming units $(\mathrm{cfu} / \mathrm{ml})$ and incubated at $25^{\circ} \mathrm{C}$ for $48-72 \mathrm{~h}$. The minimum inhibitory concentration (MIC) was the lowest concentration of the tested compound that yield no visible growth on the plates. To ensure that the solvent had no effect on the fungal growth, a control was performed with the test medium supplemented with DMSO at the same dilutions as used in the experiments. The results are incorporated in Table 2.

\section{Conclusions}

We have synthesized a series of coumarin sulfonamides bearing an azido group. The investigation of antimicrobial screening data reveals that the synthesized compounds showed significant antibacterial activity to both Gram-positive and Gram-negative species. In many cases these showed equivalent to Ciprofloxacin. On the other hand these compounds showed fungicidal activity which was higher than Fluconazole. As a result, we can say that these are better fungicidal when their antimicrobial activity is compared. Hence, it is concluded that there is ample scope for further study in developing these as good lead compounds in developing new antimicrobials.

\section{Acknowledgements}

The authors thank the University Sophisticated Instrumentation Centre (USIC), Karnatak University, Dharwad for IR, NMR and analytical data. Mr. Mahantesha Basanagouda is grateful to Karnatak University, Dharwad for providing University Research Studentship.

\section{References}

[1] K.C. Fylaktakidou, D.J. Hadjipavlou-Litina, K.E. Litinas, D.N. Nicolaides, Curr. Pharm. Design 10 (2004) 3813-3826.

[2] M.D. Ghate, M.V. Kulkarni, R. Shobha, S.Y. Kattimani, Eur. J. Med. Chem. 38 (2003) 297-304

[3] J.R. Hwu, R. Singha, S.C. Hong, Y.H. Chang, A.R. Das, I. Vliegen, E. De Clercq, J. Neyts, Antiviral. Res. 77 (2008) 157-162.

[4] V. Reutrakul, P. Leewanich, P. Tuchinda, M. Pohmakotr, T. Jaipetch, S. Sophasan T. Santisuk, Planta. Med. 69 (2003) 1048-1050.

[5] S. Sardari, Y. Mori, K. Horita, R.G. Micetich, S. Nishibe, M. Daneshtalab, Bioorg. Med. Chem. 7 (1999) 1933-1940.

[6] D. Egan, P. James, D. Cooke, R. O'Kennedy, Cancer Lett. 118 (1997) 201-211.

[7] P. Valenti, A. Rampa, M. Recanatini, A. Bisi, F. Belluti, P. Da Re, M. Carrara, L. Cima, Anticancer. Drug Des 12 (1997) 443-451.

[8] M.V. Kulkarni, G.M. Kulkarni, C.H. Lin, C.M. Sun, Curr. Med. Chem. 13 (2006) 2795-2818.

[9] K. Lewis, F.M. Ausubel, Natur. Biotech. 24 (2006) 1504-1507.

[10] M. Gellert, M.H. O'dea, T. Itoh, J.I. Tomizawa, Proc. Natl. Acad. Sci. USA 73 (1976) 4474-4478.

[11] H.S. Bevinakatti, V.V. Badiger, Arch. Pharm. (Weinheim) 314 (1981) 162-167.

[12] L. Bonsignore, F. Cottiglia, H. Elkhaili, F. Jehl, S.M. Lavagna, G. Loy, F. Manna, H. Monteil, D. Pompei, D. Secci, Il. Farmaco 53 (1998) 425-430. 
[13] K.A. Jensen, S.A.K. Christensen, Acta Chem. Scand. 3 (1947) 207.

[14] A.M. Islam, A.H. Bedair, A.A. El-Maghraby, F.M. Aly, H.A. Imam, Indian J. Chem 21B (1982) 487.

[15] S.S. Hanmantgad, M.V. Kulkarni, V.D. Patil, Indian J. Chem. 24B (1985) 459-461

[16] C.D. Lakkannavar, M.V. Kulkarni, V.D. Patil, J. Indian Chem. Soc. 69 (1992) 399-400.

[17] S.L. Born, A.S. Fix, D. Caudill, L.D. Lehmann-Mckeeman, Toxicol. Appl. Pharmacol. 151 (1998) 45-56.

[18] P. Laurin, D. Ferroud, L. Schio, M. Klich, C.D. Hamelin, P. Mavais, P. Lassaigne, A. Bonnefoy, B. Musicki, Bioorg. Med. Chem. Lett. 9 (1999) 2875-2880.

[19] M.V. Kulkarni, V.D. Patil, Arch. Pharm. (Weinheim) 314 (1981) 708-711.

[20] S.S. Hanmanthagad, M.V. Kulkarni, V.D. Patil, Ind. J. Chem. 25B (1986) 779-781.

[21] C.D. Lakkannavar, K.Y. Anklekar, G.M. Kulkarni, M.V. Kulkarni, Ind. J. Chem. 42B (2003) 1548-1550.

[22] N.S. Reddy, M.R. Mallireddigari, S. Cosenza, K. Gumireddy, S.C. Bell, E.P. Reddy, M.V. Ramana Reddy, Bioorg. Med. Chem. Lett. 14 (2004) 4093-4097.
[23] A. Purohit, L.W.L. Woo, D. Barrow, H.A.M. Hejaz, R.I. Nicholson, B.V.L. Potter, M.J. Reed, Mol. Cell. Endocrinol. 171 (2001) 129-135.

[24] N.J. Agard, J.M. Baskin, J.A. Prescher, A. Lo, C.R. Bertozzi, ACS Chem. Biol. 1 (2006) 644-648.

[25] D.H. Dube, C.R. Bertozzi, Curr. Opin. Chem. Biol. 7 (2003) 616-625.

[26] J.W. Chin, T.A. Cropp, J.C. Anderson, M. Mukherjee, Z. Zhang, P.G. Schultz, Science 301 (2003) 964-967.

[27] I.A. Khan, M.V. Kulkarni, C.M. Sun, Eur. J. Med. Chem. 40 (2005) 1168-1172.

[28] X. Hu, J. Sun, H.G. Wang, R. Manetsch, J. Am. Chem. Soc. 130 (2008) 13820-13821.

[29] E.W. Koneman, S.D. Allen, W.M. Janda, P.C. Schreckenberger, W.C. Winn. Jr., Color Atlas and Textbook of Diagnostic Microbiology, fifth ed. LippincottRaven Publishers, Philadelphia, USA, 1997, pp. 785-856.

[30] Z.K. Khan, In vitro and vivo screening techniques for bioactivity screening and evaluation. in: Proceedings of the International Workshop UNIDO-CDRI, 1997, pp. 210-211.

[31] A. Burger, G.E. Ullyot, J. Org. Chem. 12 (1947) 346. 